\title{
MULTIHYPOTHESIS MOTION COMPENSATION IN THE REDUNDANT WAVELET DOMAIN
}

\author{
Suxia Cui, Yonghui Wang, and James E. Fowler \\ Department of Electrical and Computer Engineering \\ Engineering Research Center \\ Mississippi State University, Mississippi State, MS
}

\begin{abstract}
Multihypothesis motion compensation is extended into the transform domain by using a redundant wavelet transform to produce multiple predictions that are diverse in transform phase. The corresponding inverse transform implicitly combines the multihypothesis predictions into a single spatial-domain prediction for motion compensation such that no side information is needed to describe the combination weights. Additionally, we use a hierarchical search to tailor the motion-vector field to individual phases. Substantial gains in rate-distortion performance are obtained in comparison to an equivalent system using single-phase prediction.
\end{abstract}

\section{INTRODUCTION}

Multihypothesis motion compensation (MHMC) [1] forms a prediction of pixel $s(x, y)$ in the current frame as a combination of multiple predictions in an effort to combat the uncertainty inherent in the motion-estimation process. Assuming that the combination of these hypothesis predictions is linear, we have that the prediction of $s(x, y)$ is

$$
\tilde{s}(x, y)=\sum_{i} w_{i}(x, y) \tilde{s}_{i}(x, y)
$$

where the multiple predictions $\tilde{s}_{i}(x, y)$ are combined according to some weights $w_{i}(x, y)$. A number of multihypothesis techniques for motion compensation (MC) have been proposed over the last decade. One approach to MHMC is to implement multihypothesis prediction in the spatial domain; i.e., the predictions $\tilde{s}_{i}(x, y)$ are culled from spatially distinct locations in the reference frame. Included in this class of MHMC would be fractional-pixel MC [2] and overlapped block motion compensation (OBMC) [3, 4]. Another approach is to deploy MHMC in the temporal domain by choosing predictions $\tilde{s}_{i}(x, y)$ from multiple reference frames. Examples of this class of MHMC are bidirectional prediction (Bframes) as used in MPEG-2 and H.263 and long-term-memory motion compensation (LTMMC) [5]. Of course, it is possible to combine these two classes by choosing multiple predictions that are diverse both spatially and temporally [6]. Note that the calculation of (1) in the decoder must be identical to that in the encoder; consequently, it will be necessary to transmit the weights $w_{i}(x, y)$ to the decoder as side information in the case that the weights are not fixed or not determinable from information already possessed by the decoder. Although implementation dependent, B-frames and LTMMC typically incur this additional side-information burden while fractional-pixel $\mathrm{MC}$ and $\mathrm{OBMC}$ do not.

In this paper, we propose a new class of MHMC by extending the multihypothesis-prediction concept into the transform domain. Specifically, we perform motion estimation (ME) as well as MC in the domain of a redundant, or overcomplete, wavelet transform, and use multiple predictions that are diverse in transform phase. An interesting aspect of our approach is that low-resolution information is inherently predicted with a greater number of hypotheses which corresponds to the greater difficulty inherent in estimating motion in signals with spatially low resolution. Additionally, since the weighting of the individual predictions is carried out implicitly in the form of an inverse transform, no side information need be sent to the decoder.

Below, we describe our approach in greater detail. We first provide an overview of the theory behind the redundant discrete wavelet transform (RDWT). We follow with a description of our redundant-wavelet multihypothesis (RWMH) coder that employs an RDWT to implement multihypothesis prediction. We conclude with experimental results that show a significant gain over an equivalent system [7] that employs simply single-phase prediction.

\section{THE REDUNDANT WAVELET TRANSFORM}

The RDWT [8] removes the downsampling operation from the traditional critically sampled DWT to produce an overcomplete representation. The shift variance of the DWT arises from its use of downsampling, while the RDWT is shift invariant since the spatial sampling rate is fixed across scale. As a result, the size of each subband in an RDWT is exactly the same as that of the input signal. 1D and 2D RDWTs are illustrated in Figs. 1 and 2, respectively.

By appropriately subsampling each subband of an RDWT, one can produce exactly the same coefficients as does a critically sampled DWT applied to the same input signal. In fact, in a $J$-scale 1D RDWT, there exist $2^{J}$ distinct critically sampled DWTs corresponding to the choice between even- and odd-phase subsampling at each scale of decomposition. In the algorithme à trous [8] implementation, each block of $2^{J}$ coefficients at scale $J$ of a $J$-scale 1D RDWT contains exactly one coefficient from each of these $2^{J}$ critically sampled DWTs. Since this block gathers all possible subsampling phases at this scale, we call this structure a "phase group." We can similarly define phase groups for scales $j<J$. These phase groups contain $2^{j}$ distinct phases, and there is a tree-like relationship among the phase groups at different scales. Specifically, each of the $2^{j}$ phases at scale $j$ begets two phases at scale $2^{j+1}$. This relationship is illustrated in Fig. 1 .

The situation is similar in 2D, as illustrated in Fig. 2. A $J$ scale 2D RDWT consists of $4^{J}$ distinct critically sampled DWTs, while a phase group at scale $j$ is a block of $2^{j} \times 2^{j}$ coefficients defined so as to contain all $4^{j}$ possible phases at that scale. As in the 1D transform, there is a tree-like relationship between the phases at different scales. Specifically, in the 2D RDWT, each phase at scale $j$ begets four phases at scale $j+1$.

To invert a $J$-scale 2D RDWT, one can independently invert 
each of the $4^{J}$ critically sampled DWTs constituting the RDWT and average the resulting reconstructions together. However, this implementation of the inverse RDWT incurs unnecessary duplicate synthesis filterings of the highpass bands; thus, one usually alternates between synthesis filtering and reconstruction averaging on a scale-by-scale basis in practical implementations. The final reconstruction of this practical implementation, however, is identical to that produced by the conceptually simpler DWT-based approach.

\section{REDUNDANT-WAVELET MULTIHYPOTHESIS MOTION ESTIMATION AND COMPENSATION}

Each of the critically sampled DWTs within a RDWT will view motion from a different perspective. Consequently, if motion is predicted in the RDWT domain, the inverse RDWT forms a multihypothesis prediction in the form of (1). Specifically, for a $J$-scale RDWT, the reconstruction from DWT $i$ of the RDWT is $\tilde{s}_{i}(x, y)$, $0 \leq i<4^{J}$, while $w_{i}(x, y)=4^{-J}, \forall i$. Below, we present our RWMH video-coding system that performs MHMC in precisely this fashion.

\subsection{System Architecture}

The encoder of our RWMH video-coding system is depicted in Fig. 3. The current and reference frames are transformed into RDWT coefficients, and both ME and MC take place in this redundant-wavelet domain.

In a $J$-scale RDWT decomposition, each $B \times B$ block in the original spatial domain corresponds to $3 J+1$ blocks of the same size, one in each subband. The collection of these co-located blocks is called a set. In the ME procedure, block matching is used to determine the motion of each set as a whole. Specifically, a block-matching procedure uses a cross-subband distortion measure that sums absolute differences for each block of the set similar to the cross-subband ME procedure of [7]. A window $[-W, W]$ is used for the block search, and, to speed the search, a 1-scale RDWT, rather than the full $J$-scale transform, is used for the block-matching ME procedure.

After the ME search has determined motion vectors for each set, the current- and reference-frame RDWT decompositions are continued to a total of $J$ scales. A motion-compensated frame is then created in the RDWT domain using the same motion vector for each block of the set. The inverse RDWT is performed on this RDWT-domain motion-compensated frame, combining the multiple phases into a spatial-domain multihypothesis prediction. This spatial-domain prediction is subtracted from the current frame, and the residual is coded. This final encoding step consists of a stillimage coder; for the experiments below, we use SPIHT [9], but any still-image coder, wavelet-based or otherwise, would suffice.

\subsection{Phase-Optimal Vector Search}

In the system as described above, each critically sampled DWT in the RDWT yields a different prediction of the motion of the frame, and these separate predictions are combined into a single multihypothesis prediction via the inverse-RDWT operation. However, all of the constituent DWTs use the same motion-vector field to describe the motion. More accurate prediction results when motion fields are optimized to each DWT, albeit at the expense of additional rate.

Specifically, we propose a multiscale hierarchical ME scheme which assigns to each phase at each scale a different motion-vector field. This hierarchical ME approach bears some resemblance to traditional hierarchical ME/MC; however, in our case, the hierarchy starts at high resolution and proceeds toward low resolution.
That is, we refine, for each phase at each scale, the motion vectors resulting from the block search described above starting at scale 1 and continuing to scale $J$. Consider a block of size $B \times B$ at scale 1 and call the motion vector $V$ determined for this block using the procedure above the "all-phase" motion vector. We perform a block search with a small window of $\left[-W^{\prime}, W^{\prime}\right]$ about the location indicated by the all-phase motion vector for this block. However, in the cross-subband distortion metric for this search, we include only those coefficients belonging to phase 0 ; additionally, this distortion metric is limited to only the subbands at scale 1. This search will yield a "single-phase" motion vector, $V_{1,0}$. We repeat this process for the other three phases yielding single-phase vectors $V_{1,1}, V_{1,2}$, and $V_{1,3}$. In addition to $V$, for each block, we transmit "refinement" vectors

$$
v_{1, i}=V_{1, i}-V
$$

for each phase $i$.

For scales $j>1$, we can use the vector $V_{1, i}$ for all the phases that are descendants of phase $i$ at scale 1 . Alternatively, we can apply the above procedure to further refine the motion estimate for higher scales. For example, in scale 2 , we search in a $\left[-W^{\prime}, W^{\prime}\right]$ window about $V_{1,0}$ to find the four motion vectors for the four phases at scale 2 that are children of phase 0 at scale 1 . Note that, for each additional scale of refinement, the number of additional refinement vectors that need to be sent increases by a factor of 4 -there will be 4 refinement vectors per set for one scale of refinement, 16 for two scales of refinement, etc. Fig. 4 illustrates this multiscale motion-vector refinement procedure.

The PSNR performance of the RWMH system improves as more scales of refinement vectors are used; Fig. 5 illustrates this improvement for several scales of refinement for the "Susie" sequence at a fixed rate. However, we observe diminishing returnsthe amount of PSNR improvement decreases with each additional scale of refinement. However, since the number of refinement vectors grows dramatically with each additional scale of refinement, we have concluded that the cost in rate does not justify the incremental increase in PSNR performance beyond one scale of refinement. Thus, for the experiments below, we transmit for each set of blocks one all-phase motion vector and four single-phase refinement vectors. $W^{\prime}$ is chosen so that $W^{\prime} \ll W$ in order to minimize the rate burden associated with the refinement vectors.

\section{EXPERIMENTAL RESULTS}

In our experimental results, we code grayscale sequences with the first frame intra-coded (I-frame) while all subsequent frames use ME/MC (P-frames). All wavelet transforms (DWT and RDWT) use the popular 9-7 biorthogonal filter with symmetric extension, and all ME/MC methods use integer-pixel accuracy. Since SPIHT, used as the core compression engine in all experiments, produces an embedded coding, each frame of the sequence is coded at exactly the specified target rate. The RDWT-based MHMC procedure uses $B=16, W=15$, and $W^{\prime}=1$. All rate figures include all motion-vector overhead.

We demonstrate that our proposed RWMH system yields significant performance improvement over the system of [7], which is a single-phase equivalent to our RWMH system. In the system of [7], ME is executed within the RDWT domain; however, only a single critically sampled DWT is predicted, and the ME is optimized to that single phase. Average PSNR figures for fixed bit rate are tabulated in Table 1, and frame-by-frame PSNR profiles for two sequences are shown in Figs. 6 and 7. In these results, 
"RDWT Block" refers to the system of [7]. In addition, "Spatial Block" refers to block-based ME/MC in the spatial domain, the traditional method employed in video-coding standards, followed by an entire-image DWT and then SPIHT coding of the DWT coefficients.

These results illustrate that multihypothesis prediction in the form of our RWMH system achieves at least a 0.4-dB gain over single-phase prediction. For sequences with complex motion, our RWMH system achieves even larger performance gains. For example, RWMH exhibits a gain of nearly $1 \mathrm{~dB}$ over the system of [7] for the "Football" sequence, and a gain of over $2 \mathrm{~dB}$ over the spatial-domain system.

\section{CONCLUSIONS}

In this paper, we propose the RWMH system which extends the idea of MHMC into transform domain. Recognizing that different phases in RDWT coefficients view the motion from different perspectives, we treat each critically sample DWT within the RDWT as a separate hypothesis prediction. An inverse RDWT operation implicitly combines the multiple predictions with no need for side information concerning prediction weights. Additionally, we use a hierarchical search to tailor the motion-vector field to individual phases. Substantial gains are obtained in comparison to an equivalent single-phase prediction.

\section{REFERENCES}

[1] G. J. Sullivan, "Multi-hypothesis motion compensation for low bit-rate video coding," in Proceedings of the International Conference on Acoustics, Speech, and Signal Processing, Minneapolis, MN, April 1993, vol. 5, pp. 437-440.

[2] B. Girod, "Motion-compensating prediction with fractionalpel accuracy," IEEE Transactions on Communications, vol. 41, no. 4, pp. 604-612, April 1993.

[3] S. Nogaki and M. Ohta, "An overlapped block motion compensation for high quality motion picture coding," in Proceedings of the IEEE International Symposium on Circuits and Systems, San Diego, CA, May 1992, vol. 1, pp. 184-187.

[4] M. T. Orchard and G. J. Sullivan, "Overlapped block motion compensation: An estimation-theoretic approach," IEEE Transactions on Image Processing, vol. 3, no. 5, pp. 693-699, September 1994.

[5] T. Wiegand, X. Zhang, and B. Girod, "Long-term memory motion-compensated prediction," IEEE Transactions on Circuits and Systems for Video Technology, vol. 9, no. 1, pp. 7084, February 1999.

[6] M. Flierl, T. Wiegand, and B. Girod, "Rate-constrained multihypothesis prediction for motion compensated video compression," IEEE Transactions on Circuits and Systems for Video Technology, vol. 12, no. 11, pp. 957-969, November 2002.

[7] H.-W. Park and H.-S. Kim, "Motion estimation using lowband-shift method for wavelet-based moving-picture coding," IEEE Transactions on Image Processing, vol. 9, no. 4, pp. 577-587, April 2000.

[8] M. J. Shensa, "The discrete wavelet transform: Wedding the à trous and Mallat algorithms," IEEE Transactions on Signal Processing, vol. 40, no. 10, pp. 2464-2482, October 1992.

[9] A. Said and W. A. Pearlman, "A new, fast, and efficient image codec based on set partitioning in hierarchical trees," IEEE Transactions on Circuits and Systems for Video Technology, vol. 6, no. 3, pp. 243-250, June 1996.
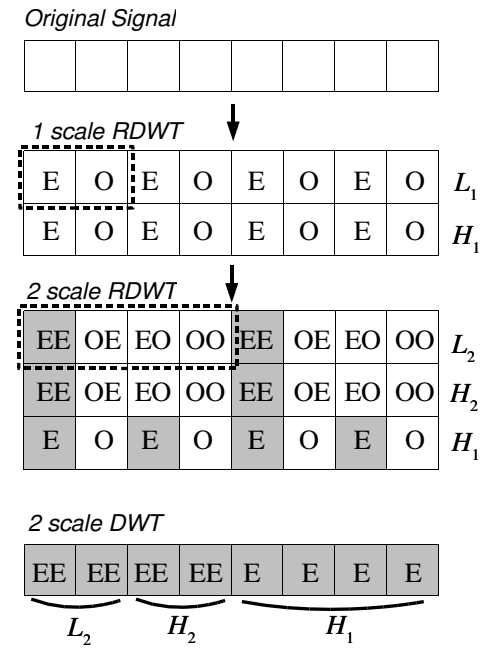

Figure 1: Relation between RDWT and critically sampled DWT. $E=$ even subsample phase, $O=$ odd subsample phase, phase groups indicated by dashed blocks. In a phase group in subband $L_{1}$, the $E$ phase begets phases $E E$ and $E O$ in subbands $L_{2}$ and $H_{2}$. A similar relationship exists for $O$ in $L_{1}$ and $O E$ and $O O$ in $L_{2}$ and $H_{2}$. Shaded coefficients indicate those constituting a 2-scale critically sampled DWT.

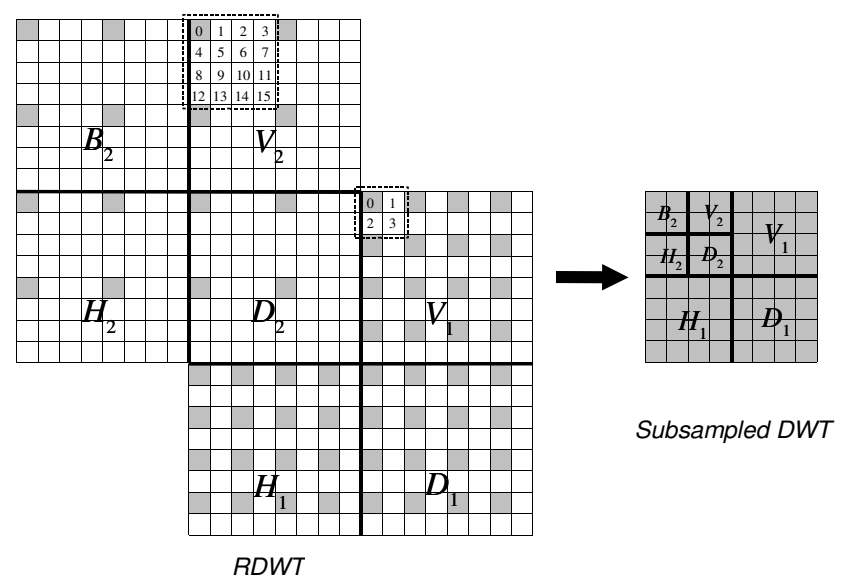

Figure 2: Phases and phase groups for a 2D RDWT. Shaded coefficients indicate those constituting a 2-scale critically sampled DWT. The phases from different scales are related in a tree. For example, phase 0 at scale 1 has phases $0,1,4$, and 5 as children at scale 2 ; phase 1 at scale 1 has a children phases $2,3,6$, and 7 at scale 2 ; etc.

\begin{tabular}{|l|c|c|c|}
\multicolumn{1}{c|}{} & \multicolumn{3}{c|}{ PSNR $(\mathrm{dB})$} \\
\cline { 2 - 4 } \multicolumn{1}{c|}{} & Spatial & RDWT & \\
Block & Block & RWMH \\
\hline Football $\dagger$ & 26.3 & 27.7 & 28.6 \\
Susie & 36.0 & 37.4 & 37.8 \\
Mother \& daughter & 40.2 & 40.8 & 41.2 \\
Coastguard & 28.1 & 28.9 & 29.5 \\
\hline
\end{tabular}

Rate is 0.25 bpp except $\dagger$, which is 0.5 bpp.

Table 1: Distortion averaged over all frames of the sequence. 


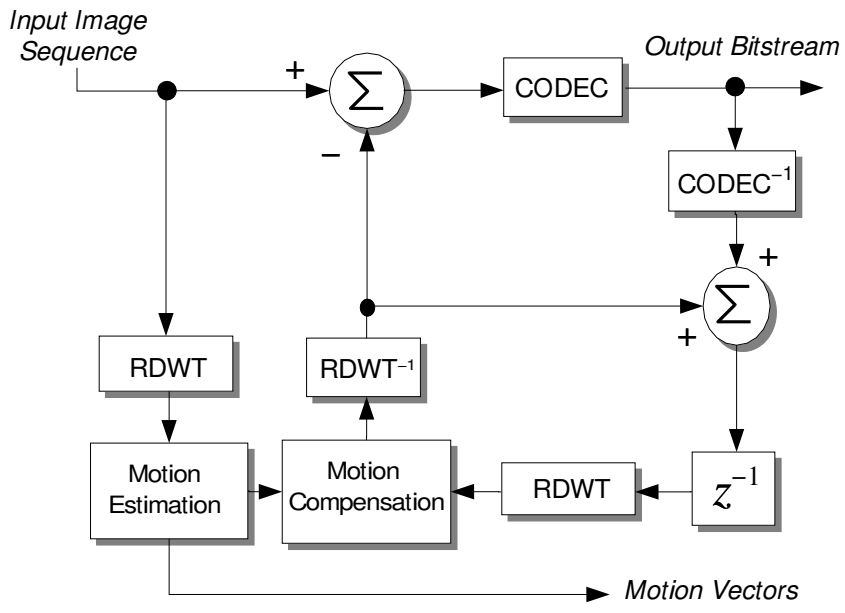

Figure 3: Block diagram of the RWMH video-coding system. CODEC is any still-image coder.

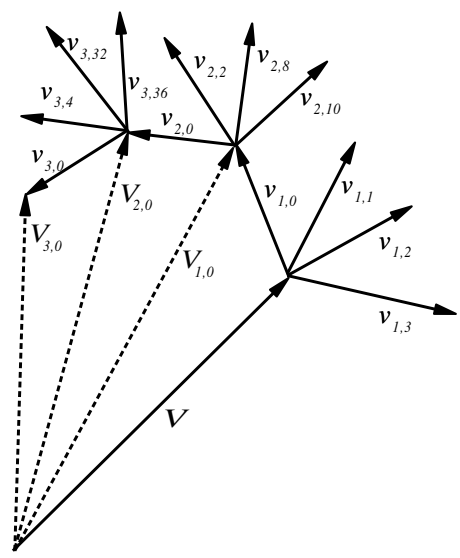

Figure 4: Hierarchical refinement of motion vectors.

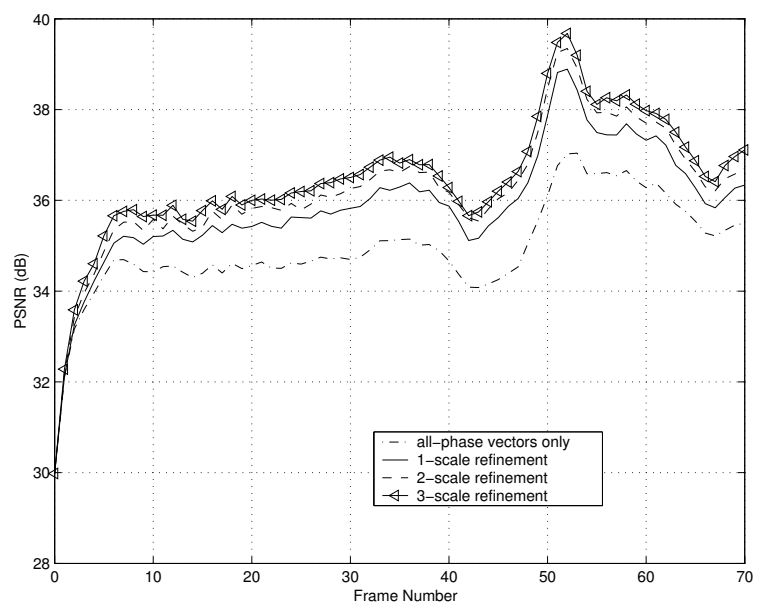

Figure 5: PSNR performance for "Susie" at $0.12 \mathrm{bpp}$ using different numbers of scales of motion-vector refinement (refinement vectors not included in the rate).

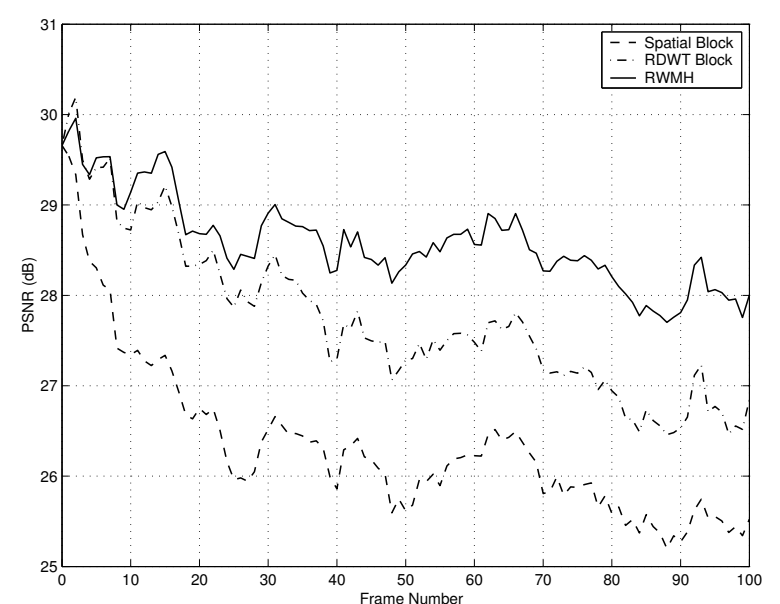

Figure 6: Frame-by-frame PSNR for "Football" at 0.5 bpp (1.3 Mbps).

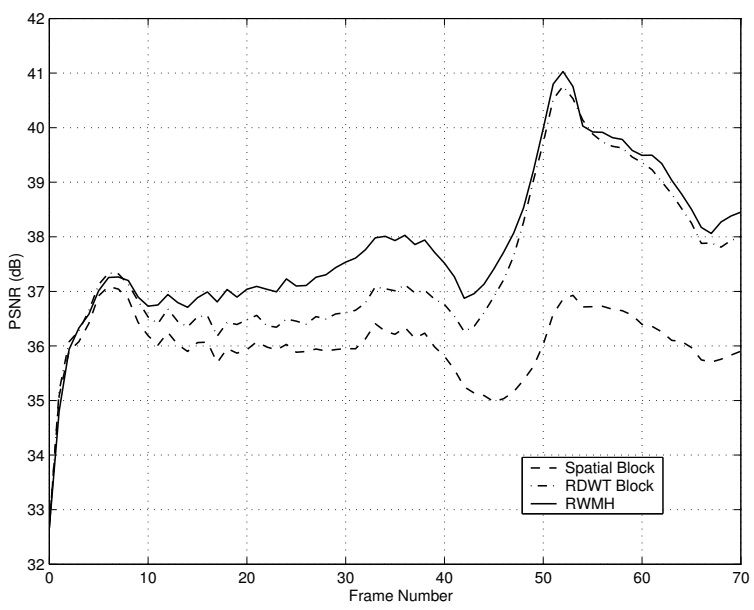

Figure 7: Frame-by-frame PSNR for "Susie" at 0.25 bpp (644 kbps). 